\title{
Very low energy implanted Bragg gratings in SOI for wafer scale testing applications
}

\author{
Renzo Loiaconoa, Rob Topleya, Agnes Nakyobea, Goran Mashanovicha, Russel Gwilliama, Giorgio Lullib, \\ Ran Feldesh', Richard Jones ${ }^{\mathrm{d}}$, Graham Reed ${ }^{\mathrm{a}}$ \\ aAdvanced Technology Institute, Faculty of Electronics and Physical Sciences, University of Surrey, Guildford, Surrey, GU2 7XH \\ bIstituto per la Microelettronica ed i Microsistemi, via Piero Gobetti, 101, 40129 Bologna, Italy \\ cNumonyx Memory Solution, Qiryat-Gat 82109, Israel \\ d Photonics Technology Labs, Intel Corporation, Mission Boulevard, Santa Clara (CA), SC12-326 \\ Contact email: R.Topley@surrey.ac.uk
}

\begin{abstract}
We present Bragg gratings with an effective index change introduced by implanting germanium at only $15 \mathrm{KeV}$. An extinction ratio of $35 \mathrm{~dB}$ at $1350 \mathrm{~nm}$ is demonstrated for device lengths of $600 \mu \mathrm{m}$, furthermore laser annealing is demonstrated.
\end{abstract}

\section{Introduction}

In the information age current technologies struggle to deliver the data rates required by modern communications and computer bus systems. Silicon photonics has the potential of overcoming some of these obstacles by relying on a well understood material system and technology [1]. However the full adoption of silicon based photonics would require complex integrated optoelectronic systems to be manufactured in high volumes at low costs. These requirements cannot be met without enabling a wafer scale testing [2] strategy in a similar fashion to what has been happening for many decades in the integrated electronics industry.

The current challenge for silicon photonics in this area is represented by the inability of the light signal to access a processed wafer without substantially modifying the wafer surface. Most of the solutions presented for light coupling into test samples rely on substantially modifying the structure of the material to enable end fire coupling, prism coupling [3], inverted tapers [4], and cantilever structures [5]. Introducing these kinds of test points, or even wavelength selective test points such as etched or metal gratings on a processed wafer can potentially introduce undesired alterations of the light propagation, such as scattering and losses, as well as interfering with successive processing steps. In order for optical wafer scale testing to become a viable technology, optical wafer scale testing should be implemented as a minimally intrusive technology. The use of ion implanted optical structures is particularly suited for these applications, as the refractive index change can be introduced on the wafer surface without altering the general topography of the wafer. Furthermore, since the planarity of the wafer is retained, it could be possible to employ these structures for applications that require extensive surface interaction such as bonding or flip chip interactions, and sensing.

The use of a low energy/low dose implant conditions $\left(10^{15} \mathrm{ion} / \mathrm{cm}^{2}\right.$, as opposed to general doping densities which can reach $10^{19} \mathrm{ion} / \mathrm{cm}^{2}$ ) potentially allows minimal optical losses to be introduced by the implantation process.

\section{Device concept and implementation}

The implanted grating device fabrication consists of four steps as shown in Figure 1. The starting material chosen is an SOI wafer with epitaxial silicon thickness of 400nm and buried oxide thickness of 1um. A 200nm thick silicon dioxide hardmask is deposited by CVD on the silicon surface and the grating pattern is defined on the hardmask by a deep UV lithography process followed by a reactive ion etching step.
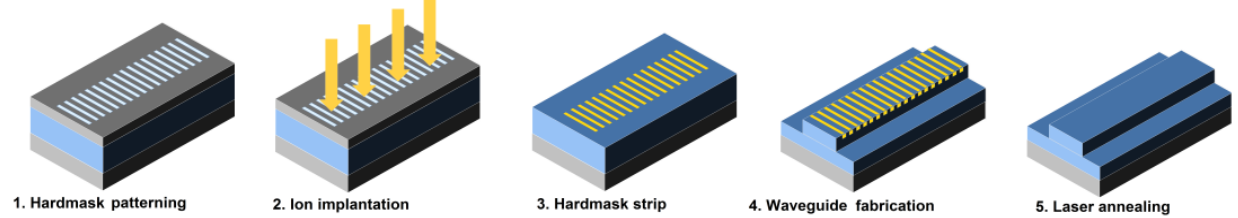

Figure 1 - Fabrication Process for Implanted Gratings

The patterned hardmask allows selective implantation of the silicon surface, transferring the grating template to the silicon. The refractive index change is caused by the silicon amorphization induced by the ion damage in the material [6]. Considering a defect accumulation model [7], in the literature the threshold of silicon to amorphous silicon transition by ion implantation has been associated with a concentration of point defects of approximately $10^{22} \mathrm{~cm}^{-3}$ [8]. It can thus be assumed that when the concentration of point defects reaches a value to $4 \times 10^{22} \mathrm{~cm}^{-3}$, which corresponds to $80 \%$ of the crystalline silicon atomic density, the material is completely amorphous. 
In this work the ion implantation conditions chosen are an ion energy of $15 \mathrm{keV}$, an ion dose of $10^{15} \mathrm{ions} / \mathrm{cm}^{2}$, and the implants were conducted at room temperature. Material amorphization has been simulated using the KING3D Monte Carlo algorithm [9]. The predicted thickness of the amorphous material is approximately $20 \mathrm{~nm}$ below the implanted surface. The use of germanium as ion specie retains CMOS compatibility and enables material amorphization without cooled implants, thus increasing the process flexibility. After the ion implantation step is complete, the hardmask layer can be reduced by a wet etch step and the waveguide structures can be defined by optical lithography over the implanted areas. The etch depth for the rib structures is $200 \mathrm{~nm}$ and the waveguide width is $500 \mathrm{~nm}$, in order to ensure single mode propagation. Furthermore, after successful device testing, the refractive index change introduced by amorphization can be reversed by excimer laser annealing [10]. In this work the etch depth for the rib structures is $200 \mathrm{~nm}$ and waveguide width is $500 \mathrm{~nm}$, in order to ensure single mode propagation.

The Bragg grating period can be designed by considering the phase matching condition $\Lambda=m \lambda_{d} / 2 n_{\text {eff }}$ [11], where $\mathrm{m}$ is the grating order, $\lambda_{\mathrm{c}}$ is the central wavelength of the stop band reflected by the grating, $\mathrm{n}_{\mathrm{eff}}$ is the effective index of the propagating mode and $\Lambda$ is the grating period.

In this work, devices were designed to enable operation at $1350 \mathrm{~nm}$ wavelengths, yielding a grating period of $220 \mathrm{~nm}$.

\section{Experimental results}

The fabricated devices were tested using an input from a DL-CS3207A super luminescent LED with a central wavelength of $1310 \mathrm{~nm}$ from Denselight [12]. The output spectrum was measured using a Hewlett Packard HP86140A optical spectrum analyser. Results are shown for a set of implanted devices with grating lengths up to $600 \mu \mathrm{m}$, with a target filtering response centred at $1350 \mathrm{~nm}(220 \mathrm{~nm}$ period), as shown in figure 2. Devices with filtering response at $1550 \mathrm{~nm}$ have also been presented elsewhere [13].
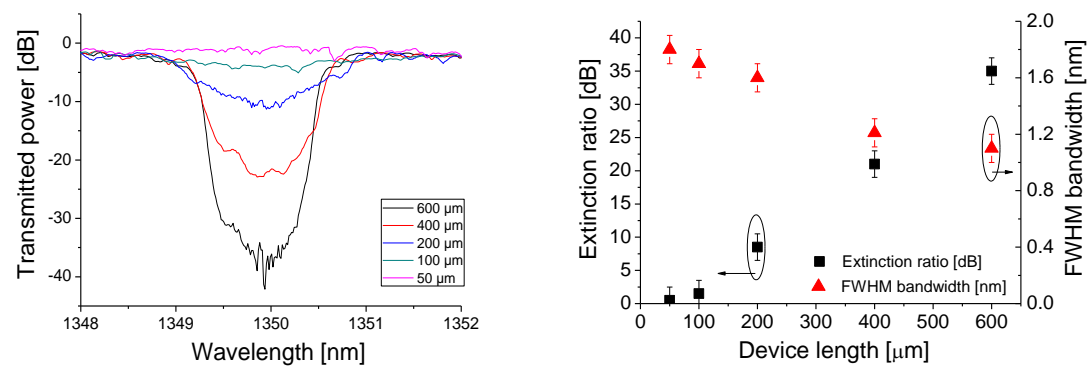

Figure 2: Transmission spectrum for $1350 \mathrm{~nm}$ central wavelength Bragg gratings with a $220 \mathrm{~nm}$ period. The duty cycle is $50 \%$, and lengths in the $50-600 \mu \mathrm{m}$ range.

The spectral response for devices of 50 and $100 \mu \mathrm{m}$ length is negligible, although a perturbation in the transmitted spectrum is noticeable at the wavelengths of interest. Devices of $200 \mu \mathrm{m}$ and $400 \mu \mathrm{m}$ in length displayed average extinction ratios of $8.5 \mathrm{~dB}$ and $21 \mathrm{~dB}$ respectively. The extinction ratio increases to $35 \mathrm{~dB}$ for the $600 \mu \mathrm{m}$ long devices. The measured extinction ratios displayed variations within $2 \mathrm{~dB}$ for devices measured on different chips from the same fabrication run. The average measured full width half maximum of the grating frequency response is $1.6 \mathrm{~nm}$ for the $200 \mu \mathrm{m}$ long device, $1.21 \mathrm{~nm}$ for the $400 \mu \mathrm{m}$ long devices, and $1.1 \mathrm{~nm}$ for the $600 \mu \mathrm{m}$ long devices. The analyzed devices produced an average measured coupling efficiency of $75 \pm 5 \mathrm{~cm}^{-1}$.

Optical losses have been measured by Fabry-Perot resonance method, implemented by using an Agilent 81689A tuneable laser. The average propagation loss value for the implanted devices was measured to be $2.2 \mathrm{dBcm}^{-1} \pm$ $0.82 \mathrm{dBcm}^{-1}$. For a $600 \mu \mathrm{m}$ long device, this corresponds to an insertion loss of only $0.13 \mathrm{~dB}$. Furthermore, devices of different lengths did not show significant differences in terms of optical losses when compared to the unimplanted waveguides. The lack of a distinctive trend in the collected loss data allows us to assume that the loss contribution associated to the ion implantation is negligible compared to the loss of the waveguide.

In order to illustrate the erasability of the implanted Bragg gratings, the implanted devices were annealed by irradiating them with pulses from a Lambda Physik LPX210 KrF excimer laser operating at $248 \mathrm{~nm}$ with pulse duration of $25 \mathrm{~ns}$ over contained areas of $3 \times 3 \mathrm{~mm}^{2}$ size. This allows recrystallizing the amorphous material by raising its temperature locally to higher than its melting point, while keeping the macroscopic portion of the wafer at room temperature, thus reversing the refractive index change previously introduced. The data presented in this section is part of larger study recently presented elsewhere [14]. The results of laser annealing at $278 \mathrm{~mJ} / \mathrm{cm}^{2}$ on the grating spectral performance is shown in figure 3-a for $30 \mathrm{keV}$ implanted gratings of $600 \mu \mathrm{m}$ length and period of $212 \mathrm{~nm}$. The extinction ratio of the grating is completely removed after annealing, therefore the data shows that it is possible 
to thermally erase implanted structures completely without compromising the waveguides. TEM cross-sections of the implanted grating before and after laser annealing are shown in the inset and in figure 3-b. The structure displays almost complete recrystallisation of the amorphous grating, although residual ion damage appears in the waveguide close to the amorphous - crystalline interface areas.
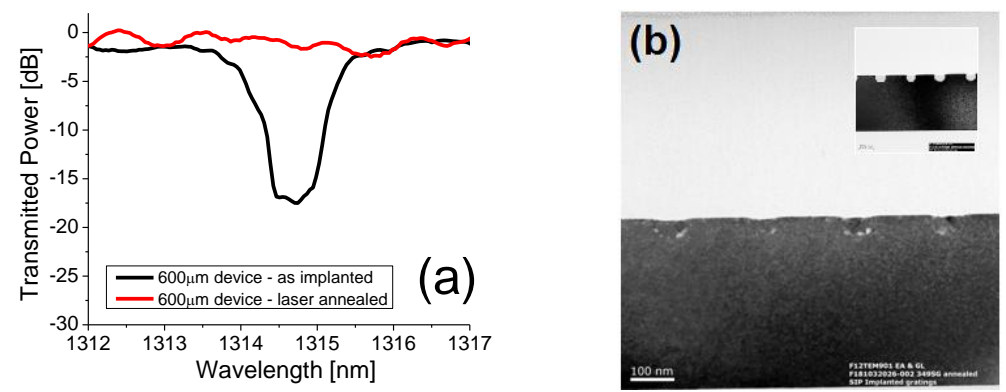

Figure 3: (a) Grating frequency response has been measured before and after laser annealing treatment for a Ge implanted grating at $30 \mathrm{keV}$ energy (b) TEM cross-sections of the implanted grating before and after laser annealing are shown in the inset and in the figure respectively.

\section{Conclusions}

Low energy (15keV) germanium implanted Bragg gratings fabricated on sub micron SOI waveguides have been demonstrated in this work. An extinction ratio of up to $35 \mathrm{~dB}$ has been demonstrated for the device length up to $600 \mu \mathrm{m}$. Loss analysis indicated that the loss contribution from the ion implantation is negligible compared to the unimplanted propagation losses, adding flexibility in implanted device applications. Furthermore, the possibility of fabricating minimally invasive implanted resonant structures in SOI waveguides may be used as a starting point to implement a wafer scale testing strategy in future silicon photonics applications. The device performance resulted to be comparable, if not higher, than etched SOI Bragg gratings presented in literature. The device design presented in this paper is also a further improvement of the work previously reported by the same authors [13]-[15], as device size and implantation energy have been reduced, whilst improving the grating performance in terms of the extinction ratio. The possibility of selectively erasing the implanted structures has been also reported. Initial testing has demonstrated that devices can be locally erased within an area of $3 \times 3 \mathrm{~mm}^{2}$, whilst leaving the unexposed area of the chip still functional. The grating technology demonstrated in this paper could be used to fabricate temporary resonant structures, allowing optical testing to be done on arbitrary areas of an optical chip

\section{References}

1. Reed, G.T., Silicon photonics : the state of the art. Chichester: Wiley. xxi, 330 p.,2008

2. L. Zimmermann, T.T., H. Schroeder, P. Dumon, and W. Bogaert, "How to bring nanophotonics to application - silicon photonics packaging". IEEE LEOS. v. 22(6),2008

3. Yi, Y., J.G. Sandland, S. Akiyama, X. Duan, and L.C. Kimerling, "Prism coupling to on-chip silicon based Bragg cladding waveguide". Applied Physics Letters. v. 85(16): p. 3381-3383,2004

4. Shoji, T., T. Tsuchizawa, T. Watanabe, K. Yamada, and H. Morita, "Low loss mode size converter from 0.3 m square Si wire waveguides to singlemode fibres". Electronics Letters. v. 38(25): p. 1669-1670,2002

5. Chen, L., C.R. Doerr, Y.-K. Chen, and T.-Y. Liow, "Low-loss and broadband cantilever couplers between standard cleaved fibers and high-index-contrast Si3 N4 or Si Waveguides". IEEE Photonics Technology Letters. v. 22(23): p. 1744-1746,2010

6. Baranova, E.C., V.M. Gusev, Y.V. Martynenko, C.V. Starinin, and I.B. Haibullin, "On silicon amorphization during different mass ion implantation". Radiation Effects. v. 18(1-2): p. 21-26,1973

7. Rimini, E., Ion Implantation: Basics to Device Fabrication. The Springer International Series in Engineering and Computer Science, ed. Springer: Springer.1994

8. Hobler, G. and G. Otto, "Status and open problems in modeling of as-implanted damage in silicon". Materials Science in Semiconductor Processing. v. 6(1-3): p. 1-14,2003

9. Lulli, G., M. Bianconi, A. Parisini, and E. Napolitani, "Structural characterization and modeling of damage accumulation in in implanted Si". Journal of Applied Physics. v. 95(1): p. 150-5,2004

10. Adikaari, A.A.D.T., N.K. Mudugamuwa, and S.R.P. Silva, "Nanocrystalline silicon solar cells from excimer laser crystallization of amorphous silicon". Solar Energy Materials and Solar Cells. v. 92: p. 634,2008

11. Yariv, A. and P. Yeh, Optical Waves in Crystal: John Wiley \& Sons. 1983

12. Dense Light. 2009 [cited; Available from: http://www.denselight.com.

13. Loiacono, R., Reed G.T., Gwilliam R. M., Mashanovich G. Z., O’Faolain L., Krauss T., Lulli G., Jeynes C., Jones R., Germanium implanted Bragg gratings in silicon on insulator waveguide, in Proc. SPIE Vol. 7606. 2010.

14. Loiacono, R., G.T. Reed, G.Z. Mashanovich, R.M. Gwilliam, Y. Hu, R. Feldesh, and R. Jones, "Laser erasable implanted gratings for integrated silicon photonics". Optics Express, accepted.

15. Loiacono, R., G.T. Reed, G.Z. Mashanovich, R.M. Gwilliam, G. Lulli, R. Feldesh, and R. Jones. Low energy silicon on insulator ion implanted gratings for optical wafer scale testing. 2011. USA: SPIE - The International Society for Optical Engineering. 